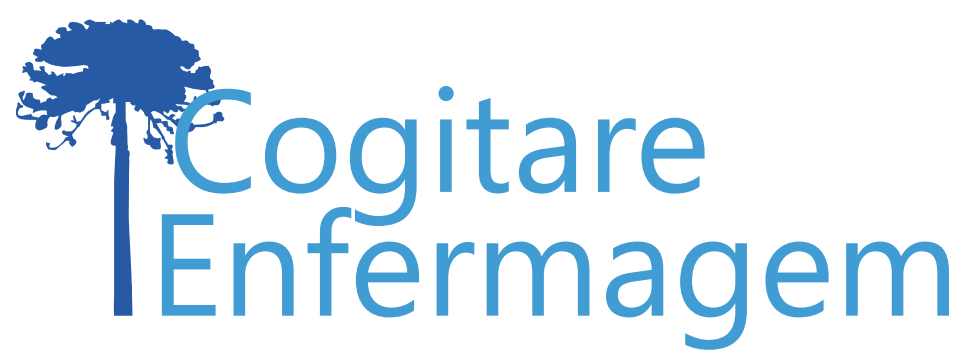

REVISÃO

\title{
ESTRATÉGIAS PARA MINIMIZAR OS RISCOS ERGONÔMICOS EM TRABALHADORES DE LIMPEZA: REVISÃO INTEGRATIVA
}

\author{
Emanuelli Mancio Ferreira da Luz $^{1} \odot$ \\ Oclaris Lopes Munhoz ${ }^{2}$ (i) \\ Bruna Xavier Morais ${ }^{2}$ () \\ Silvana Cruz da Silva ${ }^{2}$ ( \\ Franciele Ormizinda Almeida ${ }^{2} \odot$ \\ Tânia Solange Bosi de Souza Magnago² $\odot$
}

\begin{abstract}
RESUMO
Objetivo: identificar estratégias para minimização dos riscos ergonômicos em trabalhadores de limpeza.

Metodo: revisão integrativa, realizada nas bases LILACS, Scopus e MEDLINE/PUBMED, entre agosto e setembro de 2019. Os descritores "ergonomia", "serviço de limpeza" e seus sinônimos foram combinados por operadores booleanos.

Resultados: dos 21 artigos analisados, prevaleceram estudos de intervenção e classificados com nível de evidência 2 (52\%); realizados em 2012 (33,3\%), na Suécia (38\%), com trabalhadores de limpeza hospitalar $(47,6 \%)$. Evidenciou-se a utilização de estratégias de educação ergonômica, modificações nas ferramentas de trabalho, exercício aeróbico, método para avaliação de risco, protocolo de vigilância, diário de tarefas, contextualização com os fatores organizacionais e psicossociais do trabalho.

Conclusão: as estratégias evidenciadas mostraram-se eficazes e poderão contribuir, para o campo da saúde, na promoção e recuperação de acometimentos físicos dos trabalhadores de limpeza.
\end{abstract}

DESCRITORES: Saúde do Trabalhador; Ergonomia; Serviço de Limpeza; Promoção da Saúde; Revisão.

\section{ESTRATEGIAS PARA MINIMIZAR LOS RIESGOS ERGONÓMICOS EN TRABAJADORES DE LIMPIEZA: REVISIÓN INTEGRADORA}

\section{RESUMEN:}

Objetivo: identificar estrategias para la minimización de los riesgos ergonómicos en trabajadores de limpieza. Método: revisión integradora, realizada en las bases de datos LILACS, Scopus y MEDLINE/PUBMED, entre agosto y septiembre de 2019. Los descriptores "ergonomía", "servicio de limpieza" y sus sinónimos fueron combinados por operadores booleanos. Resultados: entre los 21 artículos analizados, prevalecieron los estudios de intervención clasificados con nivel de evidencia 2 (52\%); realizados en 2012 (33,3\%), en Suecia (38\%), con trabajadores de limpieza hospitalaria (47,6\%). Se evidenció la utilización de estrategias de educación ergonómica, modificaciones en las herramientas de trabajo, ejercicio aeróbico, método para la evaluación del riesgo, protocolo de vigilancia, diario de tareas, contextualización con los factores organizacionales y psicosociales del trabajo. Conclusión: las estrategias evidenciadas se mostraron eficaces y podrán contribuir, en el campo de la salud, en la promoción de la salud y recuperación de las afecciones físicas que padecen los trabajadores de limpieza.

DESCRIPTORES: Salud del Trabajador; Ergonomía; Servicio de Limpieza; Promoción de la Salud; Revisión. 
Os trabalhadores de limpeza pertencem a uma categoria por vezes esquecida no contexto laboral(1). No cenário atual, são considerados como corresponsáveis pela manutenção do ambiente seguro(2), por meio da remoção de sujeiras, detritos indesejáveis e micro-organismos presentes no ambiente, contribuindo na redução dos índices de infecções $^{(2)}$ e de acidentes de trabalho ${ }^{(1)}$.

Aatividadedelimpezatêmsidoassociadasamúltiplosriscosergonômicos, quepropiciam alteração postural e lesões ocupacionais, como os distúrbios musculoesqueléticos ${ }^{(3-18)}$. Entende-se por risco ergonômico qualquer fator que possa interferir nas características psicofisiológicas do trabalhador, causando desconforto ou afetando sua saúde, dentre eles: esforço físico, levantamento de peso, ritmo excessivo de trabalho, repetitividade de movimentos e postura inadequada de trabalho(19).

A exposição dos trabalhadores de limpeza aos riscos ergonômicos está associada ao processo de trabalho, caracterizado pelo dinamismo e rapidez nas atividades, uso de força física e levantamento manual de peso ${ }^{(11,17)}$. No entanto, as tarefas frequentes, apesar de terem ferramentas e técnicas padronizadas, não são adaptadas às necessidades psicofisiológicas dos trabalhadores, expondo-os a acidentes de trabalho, diminuição da capacidade funcional e desenvolvimento de doenças ocupacionais ${ }^{(20)}$.

Os benefícios da utilização da ergonomia incluem melhoria na qualidade de vida e saúde do trabalhador ${ }^{(2)}$, aumento da produtividade e da qualidade do serviço prestado ${ }^{(20)}$. Frente ao exposto, objetiva-se identificar na literatura as estratégias para minimização dos riscos ergonômicos em trabalhadores de limpeza.

\section{MÉTODO}

Revisão integrativa da literatura, realizada em seis etapas: identificação da temática e formulação da pergunta de pesquisa; estabelecimento de critérios de inclusão e exclusão; definição das informações a serem extraídas; avaliação; interpretação e síntese do conhecimento evidenciado ${ }^{(21)}$.

Utilizou-se a estrutura $\mathrm{PICO}^{(22)}$ para a formulação da pergunta de pesquisa. Adotouse como " $P$ " (população) os trabalhadores de limpeza; "I" (intervenção ou indicador) as estratégias e "O" (desfecho) a minimização dos riscos ergonômicos. $\mathrm{O}$ critério " $\mathrm{C}$ " (comparação) não foi aplicado. Assim, delineou-se a questão de revisão: "Quais estratégias são recomendadas para minimização dos riscos ergonômicos em trabalhadores de limpeza?".

Os artigos foram selecionados entre agosto e setembro de 2019, com busca avançada nas bases: Literatura Latino-Americana e do Caribe em Ciências da Saúde (LILACS), Medical Literature Analysis and Retrieval System Online (MEDLINE) (via US National Library of Medicine - PuBMed) e SciVerse Scopus (SCOPUS). Os descritores e seus respectivos sinônimos foram selecionados pelo DeCS e MeSH (Descritores em Ciências da Saúde e Medical Subject Headings, respectivamente) e combinados por meio dos operadores booleanos (OR/AND), com o propósito de ampliar a possibilidade de localização de estudos que respondessem à questão de revisão (Quadro 1). 
Quadro 1 - Estratégias de busca nas bases de dados LILACS, MEDLINE (via PubMed) e SCOPUS. Santa Maria, RS, Brasil, 2019

\begin{tabular}{|l|l|}
\hline BASE DE DADOS & \multicolumn{1}{|c|}{ ESTRATÉGIA DE BUSCA } \\
\hline LILACS & $\begin{array}{l}\text { (("ergonomia" OR "análise ergonomica" OR "avaliação ergonomica" OR" } \\
\text { engenharia humana" OR "ergonomia cognitiva" OR "ergonomia fisica" OR } \\
\text { "institucional ergonomia visual" OR "psicologia da engenharia")) AND (("serviço de } \\
\text { limpeza")) }\end{array}$ \\
\hline $\begin{array}{l}\text { MEDLINE (via } \\
\text { PUBMED) }\end{array}$ & $\begin{array}{l}((((\text { "ergonomics"[ MeSH Terms] OR "ergonomic analysis"[ MeSH Terms] OR } \\
\text { "ergonomic assessment"[ MeSH Terms] OR "human engineering"[ MeSH Terms] OR } \\
\text { "cognitive ergonomics"[ MeSH Terms] OR "physical ergonomics"[MeSH Terms] OR } \\
\text { ("institutional ergonomics" [MeSH Terms] OR "visual ergonomics"[ MeSH Terms] OR } \\
\text { "engineering psychology"[ MeSH Terms]) AND ("housekeeping hospital"[ MeSH } \\
\text { Terms] OR "housekeeping"[ MeSH Terms])) }\end{array}$ \\
\hline SCOPUS (Elsevier) & $\begin{array}{l}\text { (TITLE-ABS-KEY((("ergonomics" OR "ergonomic analysis" OR "ergonomic } \\
\text { assessment" OR "human engineering" OR "cognitive ergonomics" OR "physical } \\
\text { ergonomics" OR "institutional ergonomics" OR "visual ergonomics" OR } \\
\text { "engineering Psychology"))) AND (("housekeeping hospital" OR "housekeeping"))) }\end{array}$ \\
\hline
\end{tabular}

Fonte: Os autores (2019)

Incluíram-se artigos originais, em português, inglês ou espanhol, disponíveis online e que respondessem à questão de revisão. Considerou-se população do estudo: trabalhadores de limpeza hospitalar, doméstica, faxineiros e de empresas (escritórios, transportes, hotéis). Não foi utilizado recorte temporal e artigos duplicados foram considerados somente uma vez.

Como controle de qualidade, realizou-se seleção duplo-independente das produções, com posterior checagem das inconsistências e consenso entre as partes. Quando em situação de divergência, um terceiro revisor foi consultado. As produções foram exportadas para o

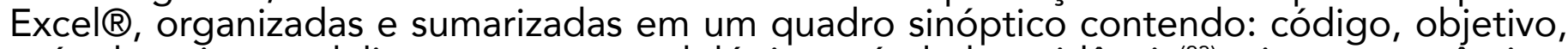
país de origem, delineamento metodológico, nível de evidência ${ }^{(23)}$, risco ergonômico, estratégia para minimização, análise ergonômica do trabalho, e avaliação e/ou resultados.

Considerou-se estratégia positiva quando proveniente de estudos experimentais e com significância estatística para a minimização dos riscos ergonômicos $(p<0,05)$ ou coeficiente kappa de Cohen $(k)$ com concordância excelente $(0,4-0,75)^{(7)}$. A estratégia foi considerada recomendada quando proveniente de estudos observacionais, visto que não possibilitam inferir causalidade.

A classificação dos níveis de evidência foi realizada conforme o tipo de questão clínica, de acordo com quatro classificações: tratamento/intervenção (avaliação de uma intervenção clínica); diagnóstico/teste (verificação de determinado fenômeno ou exposição); prognóstico, predição ou etiologia (inferir a etiologia ou a probabilidade dos resultados); e, significado (compreensão de experiências ou sentimentos em torno da doença) ${ }^{(23)}$. Os resultados estão apresentados por meio de análise descritiva. Foram respeitados os aspectos éticos, com citação fidedigna das ideias, conceitos e definições dos autores.

\section{RESULTADOS}

A partir da busca primária, foram localizadas 227 produções e, após a aplicação 
do filtro idioma, obteve-se 213 estudos. Estes foram submetidos à leitura dos títulos e resumos, com a exclusão de 186 e resultando em 27 estudos. Posteriormente, foi realizada a leitura das produções na íntegra e o corpus da pesquisa foi constituído por 21 artigos. A exclusão de artigos por indisponibilidade de maneira gratuita foi realizada depois de esgotadas as possibilidades de obtenção nos diferentes sítios eletrônicos de acesso aberto à internet (Figura 1).

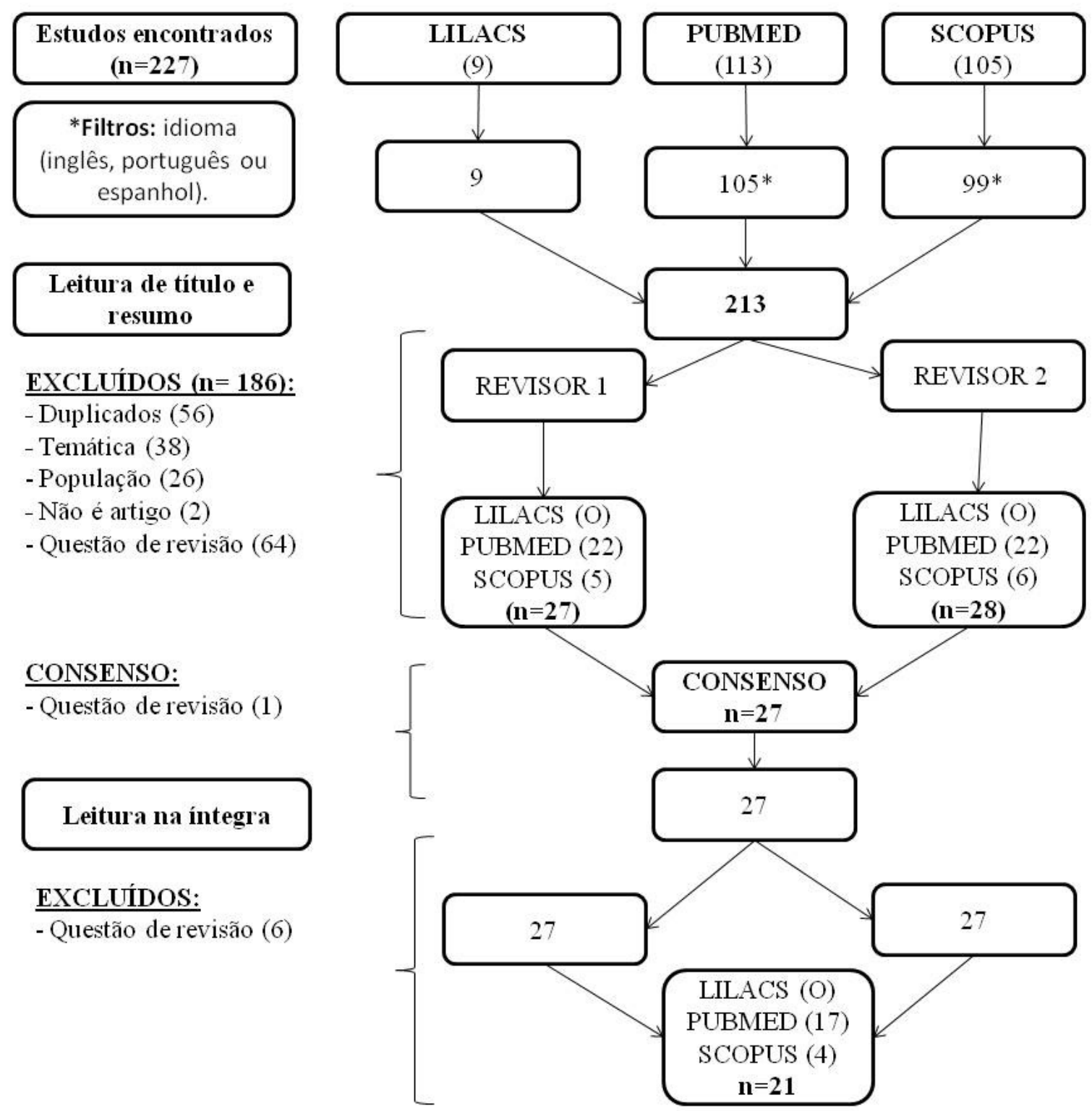

Figura 1 - Fluxograma das etapas de seleção duplo-independente das produções para compor o corpus final $(n=21)$. Santa Maria, RS, Brasil, 2019

O corpus de análise foi composto por 21 artigos, publicados entre 1998 e 2018, com destaque para 2012 (sete, 33,3\%). A Suécia teve maior número de publicações (oito, 38\%). Quanto à população, sobressaíram-se estudos com trabalhadores de limpeza hospitalar $(n=10 ; 47,6 \%)$ e de empresas (sete; 33,3\%). As estratégias recomendadas estão descritas no Quadro 2 e Quadro 3. 
Quadro 2 - Caracterização do corpus de análise da revisão integrativa, segundo objetivo, país de origem, delineamento e nível de evidência. Santa Maria, RS, Brasil, 2019 (continua)

\begin{tabular}{|c|c|c|c|}
\hline Código & Objetivo & $\begin{array}{l}\text { País de } \\
\text { origem }\end{array}$ & Delineamento e NE* \\
\hline $\mathrm{A} 1^{(3)}$ & $\begin{array}{l}\text { Comparar análises para autorregistro da elevação do } \\
\text { braço }\end{array}$ & Suécia & $\begin{array}{l}\text { Ensaio clínico randomizado } \\
\mathrm{NE}=2^{\star}\end{array}$ \\
\hline$A 2^{(4)}$ & $\begin{array}{l}\text { Investigar a contribuição da intervenção preventiva na } \\
\text { ausência de doença na empresa }\end{array}$ & Suécia & $\begin{array}{l}\text { Quase experimental } \\
\qquad \mathrm{NE}=3^{\star}\end{array}$ \\
\hline$A 3^{(25)}$ & Comparar a eficácia do exercício aeróbico no trabalho & Dinamarca & $\begin{array}{c}\text { Ensaio clínico randomizado } \\
\mathrm{NE}=2^{\star}\end{array}$ \\
\hline $\mathrm{A} 4^{(29)}$ & $\begin{array}{l}\text { Analisar o efeito do gênero nas estratégias de equilíbrio } \\
\text { trabalho/família e horários de trabalho }\end{array}$ & Canadá & $\begin{array}{l}\text { Métodos mistos } \\
\quad \mathrm{NE}=6^{\star \star}\end{array}$ \\
\hline$A 5^{(5)}$ & $\begin{array}{l}\text { Investigar o exercício aeróbico para diminuição da dor } \\
\text { musculoesquelética após } 4 \text { e } 12 \text { meses }\end{array}$ & Dinamarca & $\begin{array}{l}\text { Ensaio clínico randomizado } \\
\mathrm{NE}=2^{\star}\end{array}$ \\
\hline$A 6^{(26)}$ & $\begin{array}{l}\text { Comparar os efeitos da altura do cabo do esfregão na } \\
\text { atividade muscular do ombro e no esforço percebido }\end{array}$ & Finlândia & $\begin{array}{c}\text { Ensaio clínico randomizado } \\
\mathrm{NE}=2^{\star}\end{array}$ \\
\hline$A 7^{(6)}$ & $\begin{array}{l}\text { Avaliar problemas ergonômicos no trabalho físico e a } \\
\text { associação com o design das ferramentas }\end{array}$ & Suécia & $\begin{array}{c}\text { Ensaio clínico randomizado } \\
\mathrm{NE}=2^{\star}\end{array}$ \\
\hline$A 8^{(7)}$ & Validar um protocolo de vigilância sanitária para DORT+ & Suécia & $\begin{array}{c}\text { Ensaio clínico randomizado } \\
\qquad \mathrm{NE}=2^{\star}\end{array}$ \\
\hline$A 9^{(8)}$ & $\begin{array}{l}\text { Explorar a prevalência de sintomas osteomusculares, a } \\
\text { interrupção das atividades normais e a associação com } \\
\text { fatores de risco ergonômico }\end{array}$ & Bangladesh & $\begin{array}{l}\text { Transversal } \\
\mathrm{NE}=4^{\star \star \star}\end{array}$ \\
\hline $\mathrm{A} 10^{(9)}$ & $\begin{array}{l}\text { Comparar a eficácia da educação ergonômica na } \\
\text { atividade muscular, carga postural e cardiovascular } \\
\text { durante a limpeza }\end{array}$ & Dinamarca & $\begin{array}{l}\text { Ensaio clínico randomizado } \\
\qquad \mathrm{NE}=2^{\star}\end{array}$ \\
\hline $\mathrm{A} 11^{(10)}$ & $\begin{array}{l}\text { Examinar cobertura ativa, conformidade regulatória, } \\
\text { frequência e gravidade das perdas por lesões } \\
\text { ocupacionais }\end{array}$ & $\begin{array}{l}\text { Estados } \\
\text { Unidos da } \\
\text { América }\end{array}$ & $\begin{array}{l}\text { Estudo prospectivo } \\
\qquad \mathrm{NE}=6^{\star}\end{array}$ \\
\hline $\mathrm{A} 12^{(11)}$ & $\begin{array}{l}\text { Identificar a associação entre as atividades laborais e } \\
\text { DME§ }\end{array}$ & Brasil & $\begin{array}{c}\text { Transversal } \\
\mathrm{NE}=6^{*}\end{array}$ \\
\hline$A 13^{(12)}$ & $\begin{array}{l}\text { Investigar a relação entre DORT+, atividade muscular e } \\
\text { carga postural }\end{array}$ & Dinamarca & $\begin{array}{c}\text { Ensaio clinico randomizado } \\
\mathrm{NE}=2^{\star}\end{array}$ \\
\hline $\mathrm{A} 14^{(24)}$ & $\begin{array}{l}\text { Analisar problemas com a implementação e uso de } \\
\text { ferramentas ergonômicas e técnicas no processo de } \\
\text { limpeza }\end{array}$ & Suécia & $\begin{array}{l}\text { Ensaio clínico } \\
\text { randomizado. } \\
\qquad \mathrm{NE}=2^{\star}\end{array}$ \\
\hline$A 15^{(13)}$ & $\begin{array}{l}\text { Analisar o risco de DME§ nos membros superiores } \\
\text { durante a aspiração }\end{array}$ & Austrália & $\begin{array}{c}\text { Transversal } \\
\mathrm{NE}=6^{\star}\end{array}$ \\
\hline $\mathrm{A} 16^{(14)}$ & $\begin{array}{l}\text { Comparar a exposição ao trabalho mecânico baseada } \\
\text { em tarefas com ocupação }\end{array}$ & Suécia & $\begin{array}{c}\text { Ensaio clínico randomizado } \\
\mathrm{NE}=2^{\star}\end{array}$ \\
\hline$A 17^{(15)}$ & $\begin{array}{l}\text { Analisar a validade dos relatórios autoavaliativos de } \\
\text { ocorrência e duração das tarefas }\end{array}$ & Suécia & $\begin{array}{l}\text { Métodos mistos } \\
\quad \mathrm{NE}=6^{\star}\end{array}$ \\
\hline$A 18^{(16)}$ & $\begin{array}{l}\text { Avaliar a prevalência e fatores associados à DORT+ em } \\
\text { trabalhadores de limpeza em um hospital privado }\end{array}$ & Índia & $\begin{array}{c}\text { Transversal } \\
\mathrm{NE}=4^{\star \star \star}\end{array}$ \\
\hline$A 19^{(17)}$ & $\begin{array}{l}\text { Analisar a saúde ocupacional de limpadores brasileiros } \\
\text { em Massachusetts }\end{array}$ & $\begin{array}{l}\text { Estados } \\
\text { Unidos da } \\
\text { América }\end{array}$ & $\begin{array}{c}\text { Transversal } \\
\mathrm{NE}=6^{\star}\end{array}$ \\
\hline $\mathrm{A} 2 \mathrm{O}^{(18)}$ & $\begin{array}{l}\text { Analisar as diferenças na carga física de trabalho, fatores } \\
\text { psicossociais e DME§ com os fatores organizacionais do } \\
\text { trabalho }\end{array}$ & Suécia & $\begin{array}{l}\text { Ensaio clínico randomizado } \\
\qquad \mathrm{NE}=2^{\star}\end{array}$ \\
\hline
\end{tabular}



gênero entre trabalhadores de limpeza hospitalar

*Nível de evidência com base na questão clínica de tratamento/intervenção(23). **Nível de evidência com base na questão clínica de diagnóstico/teste ${ }^{(23)}$. ${ }^{\star \star \star}$ Nível de evidência com base na questão clínica de prognóstico/etiologiaa ${ }^{(23)}$. +Doença osteomuscular relacionada ao trabalho. §Dor musculoesquelética. (k) Coeficiente kappa de Cohen.

Fonte: Os autores (2019)

Quadro 3 - Caracterização do corpus de análise da revisão integrativa, segundo risco ergonômico, estratégia, análise ergonômica do trabalho e avaliação e resultados. Santa Maria, RS, Brasil, 2019 (continua)

\begin{tabular}{|c|c|c|c|c|}
\hline Código & $\begin{array}{l}\text { Risco } \\
\text { ergonômico }\end{array}$ & Estratégia & $\begin{array}{l}\text { Análise } \\
\text { ergonômica do } \\
\text { trabalho } \\
\end{array}$ & Avaliação e resultados \\
\hline$A 1^{(3)}$ & $\begin{array}{l}\text { Movimento de } \\
\text { elevação do } \\
\text { braço/DORT+ }\end{array}$ & $\begin{array}{l}\text { Método para } \\
\text { avaliação de risco } \\
\text { (níveis de ação) }\end{array}$ & $\begin{array}{l}\text { Protocolo de } \\
\text { vigilância em } \\
\text { condições } \\
\text { adversas de } \\
\text { ergonomia, } \\
\text { acelerômetro } \\
\text { triaxial e } \\
\text { autogravação } \\
\text { da elevação e } \\
\text { velocidade do } \\
\text { braço } \\
\end{array}$ & $\begin{array}{l}\text { A estratégia foi positiva: } \\
\text { autogravações, em combinação } \\
\text { com níveis de ação, fornecem aos } \\
\text { empregadores um método para } \\
\text { avaliação de riscos como base sólida } \\
\text { para a prevenção de DORT+. No } \\
\text { grupo controle, houve diferença entre } \\
\text { as duas referências da elevação do } \\
\text { braço de } 9^{\circ}(p<0,05)\end{array}$ \\
\hline$A 2^{(4)}$ & $\begin{array}{l}\text { Esforço físico e } \\
\text { DORT+ }\end{array}$ & $\begin{array}{l}\text { Educação } \\
\text { ergonômica, } \\
\text { medidas } \\
\text { organizacionais, } \\
\text { desenvolvimento } \\
\text { de competências, } \\
\text { medidas } \\
\text { ambientais de } \\
\text { trabalho físico e } \\
\text { psicossocial }\end{array}$ & $\begin{array}{l}\text { Protocolo } \\
\text { sobre custos e } \\
\text { contabilidade de } \\
\text { recursos humanos }\end{array}$ & $\begin{array}{l}\text { A estratégia foi positiva com } \\
\text { contribuição líquida calculada de } \\
605,6 \text { Euros }\end{array}$ \\
\hline$A 3^{(25)}$ & Esforço físico & Exercício aeróbico & $\begin{array}{l}\text { Eletromiografia de } \\
\text { superfície }\end{array}$ & $\begin{array}{l}\text { A estratégia foi positiva para maior } \\
\text { capacidade para o trabalho de } \\
0,59 \pm 0,27 \text { (IC } 95 \% ; 0,05-1,13, \\
p=0,03) \text { e diminuiu a necessidade de } \\
\text { recuperação significativamente em } \\
-11,0 \text {. } \\
\end{array}$ \\
\hline $\mathrm{A} 4^{(29)}$ & $\begin{array}{l}\text { Fatores } \\
\text { psicossociais: } \\
\text { desigualdade de } \\
\text { gênero e turno } \\
\text { de trabalho } \\
\text { restrito }\end{array}$ & $\begin{array}{l}\text { Estratégia } \\
\text { de equilíbrio } \\
\text { trabalho/ família }\end{array}$ & $\begin{array}{l}\text { Observação, } \\
\text { entrevista e diário } \\
\text { de tarefas }\end{array}$ & $\begin{array}{l}\text { Recomenda-se a criação de espaços } \\
\text { de discussão para o equilíbrio entre } \\
\text { trabalho e família, a circulação } \\
\text { equitativa de informações para reduzir } \\
\text { as desigualdades associadas a gênero/ } \\
\text { status social e as responsabilidades } \\
\text { familiares }\end{array}$ \\
\hline$A 5^{(5)}$ & $\begin{array}{l}\text { Esforço físico } \\
\text { muscular e DME§ }\end{array}$ & $\begin{array}{l}\text { Exercício aeróbico } \\
\text { e palestras de } \\
\text { promoção à saúde }\end{array}$ & Questionário & $\begin{array}{l}\text { A estratégia de exercício aeróbico, } \\
\text { comparada a palestras, foi positiva na } \\
\text { redução ( }>30 \%) \text { da intensidade da dor } \\
\text { no pescoço, ombros, braços/ pulsos } \\
\text { nos } 12 \text { meses de acompanhamento } \\
\text { ( } p=0,07) \text {. No entanto, aumentou a dor } \\
\text { nas extremidades inferiores (joelhos) }\end{array}$ \\
\hline
\end{tabular}




\begin{tabular}{|c|c|c|c|c|}
\hline$A 6^{(26)}$ & $\begin{array}{l}\text { Esforço físico } \\
\text { muscular do } \\
\text { ombro }\end{array}$ & $\begin{array}{l}\text { Modificações de } \\
\text { ferramentas de } \\
\text { trabalho }\end{array}$ & $\begin{array}{l}\text { Eletromiografia de } \\
\text { superfície }\end{array}$ & $\begin{array}{l}\text { A estratégia foi positiva: a altura do } \\
\text { cabo do esfregão ajustado teve um } \\
\text { efeito estatisticamente significativo } \\
\text { ( } p<0,001 \text { ) sobre a atividade muscular } \\
\text { do ombro ou do queixo, em } \\
\text { comparação com o nível dos olhos }\end{array}$ \\
\hline$A 7^{(6)}$ & $\begin{array}{l}\text { Desenho das } \\
\text { ferramentas } \\
\text { de trabalho e } \\
\text { DORT+ } \\
\end{array}$ & $\begin{array}{l}\text { Modificações de } \\
\text { ferramentas de } \\
\text { trabalho }\end{array}$ & Observação & $\begin{array}{l}\text { A estratégia foi positiva: utilização } \\
\text { de ferramentas com melhor design } \\
\text { ergonômico, de eixo longo ou um } \\
\text { carrinho de mão }(p<0,001)\end{array}$ \\
\hline$A 8^{(7)}$ & $\begin{array}{l}\text { DORT+ em } \\
\text { região do } \\
\text { pescoço / } \\
\text { ombros. }\end{array}$ & $\begin{array}{l}\text { Protocolo de } \\
\text { vigilância sanitária }\end{array}$ & $\begin{array}{l}\text { Procotolo } \\
\text { Vigilância Sanitária } \\
\text { em Ergonomia } \\
\text { Adversa (HECO) e } \\
\text { exame físico } \\
\end{array}$ & $\begin{array}{l}\text { A estratégia foi positiva: efetividade } \\
\text { do protocolo HECO para um ou mais } \\
\text { diagnósticos no pescoço }(86 \%, k 1 / 4 \\
0,62) / \text { ombros e cotovelos } / \text { mãos }(84 \% \text {, } \\
k 1 / 40,49)\end{array}$ \\
\hline$A 9^{(8)}$ & $\begin{array}{l}\text { Esforço físico } \\
\text { e sintomas } \\
\text { osteomusculares } \\
\text { em membros } \\
\text { superiores }\end{array}$ & $\begin{array}{l}\text { Prevenção: } \\
\text { diagnóstico } \\
\text { e atenção } \\
\text { ergonômica }\end{array}$ & $\begin{array}{l}\text { Questionário } \\
\text { Nórdico }\end{array}$ & $\begin{array}{l}\text { Recomenda-se a promoção da } \\
\text { atenção ergonômica e a interrupção } \\
\text { de atividades regulares }\end{array}$ \\
\hline $\mathrm{A} 10^{(9)}$ & $\begin{array}{l}\text { Esforço físico, } \\
\text { carga de trabalho } \\
\text { (postural e } \\
\text { cardiovascular) }\end{array}$ & $\begin{array}{l}\text { Educação } \\
\text { ergonômica }\end{array}$ & $\begin{array}{l}\text { Observação, } \\
\text { eletromiografia } \\
\text { de superfície, } \\
\text { eletrocardiograma }\end{array}$ & $\begin{array}{l}\text { A estratégia foi positiva para um } \\
\text { menor nível de atividade muscular do } \\
\text { trapézio }(p=0,03) \text {, menor amplitude de } \\
\text { movimento e velocidade angular do } \\
\text { tronco e menor carga cardiovascular } \\
(p=0,02)\end{array}$ \\
\hline $\mathrm{A} 11^{(10)}$ & $\begin{array}{l}\text { Lesões } \\
\text { ocupacionais, } \\
\text { tensão, entorse, } \\
\text { elevação e queda }\end{array}$ & $\begin{array}{l}\text { Educação } \\
\text { ergonômica }\end{array}$ & $\begin{array}{l}\text { Inspeção e } \\
\text { entrevista }\end{array}$ & $\begin{array}{l}\text { Recomendam-se programas } \\
\text { participativos educativos para } \\
\text { eliminação de perigos; contratação de } \\
\text { ergonomista e protocolos }\end{array}$ \\
\hline $\mathrm{A} 12^{(11)}$ & $\begin{array}{l}\text { DME§, má } \\
\text { postura, } \\
\text { transporte } \\
\text { manual de cargas } \\
\text { e esforço físico } \\
\end{array}$ & $\begin{array}{l}\text { Modificações na } \\
\text { organização do } \\
\text { trabalho }\end{array}$ & $\begin{array}{l}\text { Questionário sobre } \\
\text { a avaliação geral } \\
\text { de riscos }\end{array}$ & $\begin{array}{l}\text { Recomenda-se diminuir o peso } \\
\text { dos carrinhos, mantendo áreas de } \\
\text { reposição de material em cada andar }\end{array}$ \\
\hline $\mathrm{A} 13^{(12)}$ & $\begin{array}{l}\text { Carga postural, } \\
\text { atividade } \\
\text { muscular } \\
\text { e DORT+ em } \\
\text { braços e ombro }\end{array}$ & $\begin{array}{l}\text { Educação } \\
\text { ergonômica com } \\
\text { gravações }\end{array}$ & $\begin{array}{l}\text { Eletromiografia } \\
\text { de superfície, } \\
\text { acelerômetro e } \\
\text { o nível de dor } \\
\text { percebida }\end{array}$ & $\begin{array}{l}\text { A estratégia foi positiva: redução da } \\
\text { ocorrência de DORT no ângulo de } \\
\text { flexão lateral }(p<0,05) \text { Trabalhadores } \\
\text { com baixo nível de dor exibiram maior } \\
\text { atividade muscular durante a limpeza } \\
(p<0,05)\end{array}$ \\
\hline$A 14^{(24)}$ & Esforço físico & $\begin{array}{l}\text { Modificações de } \\
\text { ferramentas de } \\
\text { trabalho }\end{array}$ & $\begin{array}{l}\text { Eletromiografia } \\
\text { de superfície, } \\
\text { medidas } \\
\text { autorreferidas de } \\
\text { nível de conforto, } \\
\text { esforço percebido } \\
\text { (Escala de Borg) e } \\
\text { entrevistas } \\
\end{array}$ & $\begin{array}{l}\text { A estratégia foi positiva: diminuição } \\
\text { da atividade muscular durante o uso } \\
\text { da alça de esfregão ajustável, em } \\
\text { comparação com o esfregão não } \\
\text { ajustável: para o músculo deltoide } \\
\text { direito }(p=0,003) \text {, músculo trapézio } \\
\text { direito }(p=0,000) \text { e extensores de } \\
\text { punho esquerdo ( } p=0,004)\end{array}$ \\
\hline $\mathrm{A} 15^{(13)}$ & $\begin{array}{l}\text { Esforço físico } \\
\text { repetitivo e DME } \\
\text { nos membros } \\
\text { superiores }\end{array}$ & $\begin{array}{l}\text { Modificações de } \\
\text { ferramentas de } \\
\text { trabalho }\end{array}$ & $\begin{array}{l}\text { Filmagem; } \\
\text { avaliação de risco, } \\
\text { avaliação rápida } \\
\text { dos membros } \\
\text { superiores (RULA) }\end{array}$ & $\begin{array}{l}\text { Recomenda-se a utilização do } \\
\text { aspirador em máquina do tipo mochila } \\
\text { ( } p=0,016), \text { comparado ao do tipo } \\
\text { caixa }(p=0,011) \text {, com maior risco de } \\
\text { desenvolver DME. }\end{array}$ \\
\hline
\end{tabular}




\begin{tabular}{|c|c|c|c|c|}
\hline $\mathrm{A} 16^{(14)}$ & $\begin{array}{l}\text { Fatores } \\
\text { organizacionais, } \\
\text { trabalho } \\
\text { mecânico e DME }\end{array}$ & $\begin{array}{l}\text { Exposição ao } \\
\text { trabalho em } \\
\text { tarefas e baseadas } \\
\text { em ocupação } \\
\text { (tempo de } \\
\text { trabalho e pausa) } \\
\end{array}$ & $\begin{array}{l}\text { Eletromiografia } \\
\text { de superfície e } \\
\text { observação }\end{array}$ & $\begin{array}{l}\text { A estratégia foi positiva: o intervalo/ } \\
\text { pausa mostrou-se significativo para } \\
\text { os dois grupos na redução da DME } \\
(p=0,002)\end{array}$ \\
\hline$A 17^{(15)}$ & $\begin{array}{l}\text { Carga física } \\
\text { de trabalho e } \\
\text { DORT+ }\end{array}$ & Diário de tarefas & \begin{tabular}{|l} 
Entrevista e \\
observação
\end{tabular} & $\begin{array}{l}\text { Disparidade entre o número de } \\
\text { tarefas relatadas e observadas, a } \\
\text { subestimação da duração das pausas e } \\
\text { das atividades menos demoradas }\end{array}$ \\
\hline $\mathrm{A} 18^{(16)}$ & DORT+ & $\begin{array}{l}\text { Educação } \\
\text { ergonômica }\end{array}$ & $\begin{array}{l}\text { Questionário } \\
\text { Nórdico }\end{array}$ & $\begin{array}{l}\text { Associação significativa entre DORT e } \\
\text { aumento da duração das horas e anos } \\
\text { de trabalho }(p<0,05) \text {. Recomenda-se } \\
\text { a educação ergonômica, rotação do } \\
\text { trabalho e fisioterapia }\end{array}$ \\
\hline $\mathrm{A} 19^{(17)}$ & $\begin{array}{l}\text { Posturas } \\
\text { de trabalho } \\
\text { desconfortáveis, } \\
\text { levantamento de } \\
\text { objetos e DME }\end{array}$ & $\begin{array}{l}\text { Modificações na } \\
\text { organização e } \\
\text { ferramentas de } \\
\text { trabalho }\end{array}$ & Questionário & $\begin{array}{l}\text { Recomenda-se a utilização de } \\
\text { equipamentos ergonômicos, } \\
\text { equipamento de proteção confortável } \\
\text { (sapatos) e organização do trabalho } \\
\text { (rotação de tarefas, diminuição da } \\
\text { carga de trabalho, pausas e melhoria } \\
\text { nas relações sociais) }\end{array}$ \\
\hline $\mathrm{A} 2 \mathrm{O}^{(18)}$ & $\begin{array}{l}\text { Esforço físico, } \\
\text { carga de } \\
\text { trabalho, fatores } \\
\text { psicossociais e } \\
\text { DME§ }\end{array}$ & $\begin{array}{l}\text { Fatores } \\
\text { organizacionais do } \\
\text { trabalho }\end{array}$ & $\begin{array}{l}\text { Questionário } \\
\text { Nórdico; avaliação } \\
\text { médica e de } \\
\text { fisioterapeuta; } \\
\text { pedômetro; sensor } \\
\text { de inclinação; } \\
\text { frequência } \\
\text { cardíaca; } \\
\text { eletromiografia de } \\
\text { superfície }\end{array}$ & $\begin{array}{l}\text { A estratégia foi positiva: o grupo com } \\
\text { organização (intervenção) apresentou } \\
\text { menor carga física, frequência } \\
\text { cardíaca, posições e movimentos da } \\
\text { cabeça, dos braços }(p<0,001) \text { e do } \\
\text { punho }(p=0,001) \text { do que o grupo } \\
\text { controle. A prevalência de DME } \$ \text { no } \\
\text { pescoço/ombros foi menor no grupo } \\
\text { intervenção }(p=0,04)\end{array}$ \\
\hline $\mathrm{A} 21^{(27)}$ & $\begin{array}{l}\text { Fatores } \\
\text { organizacionais e } \\
\text { carga de trabalho }\end{array}$ & $\begin{array}{l}\text { Exposição ao } \\
\text { trabalho leve e } \\
\text { pesado e dividido } \\
\text { por gênero }\end{array}$ & $\begin{array}{l}\text { Observação e } \\
\text { entrevista }\end{array}$ & $\begin{array}{l}\text { Recomenda-se a atribuição de tarefas } \\
\text { por sexo para demandas de trabalho } \\
\text { excessivas, que seriam melhor } \\
\text { abordadas pelo redesenho de tarefas }\end{array}$ \\
\hline
\end{tabular}

Fonte: Os autores (2019)

Os riscos ergonômicos prevalentes foram relacionados ao esforço físico (8-9,11,13,18,24-26) (oito; 43\%) e sintomas musculoesqueléticos (dor osteomuscular relacionada ao trabalho/ DORT, dor musculoesquelética/DME) $)^{(3-8,10-18,26)}(n=15 ; 71,4 \%)$.

Nos ensaios clínicos ${ }^{(3,5-7,9,12,14,18,24-26)}$ e no estudo quase experimental(4), identificouse a eficácia das estratégias para minimização dos riscos ergonômicos. Dentre essas, prevaleceram e foram eficazes: a educação ergonômica $(4,9,10,12,16)$ (cinco; $23,8 \%)$; as modificações de ferramentas de trabalho ${ }^{(6,11,13,17,24,26)}$ (seis; $28,5 \%$ ) e a realização de exercício aeróbico $^{(5,25)}$ (dois; 9,5\%).

Nos estudos transversais ${ }^{(8-9,11,13,16-17)}$ e prospectivo(10), foi identificada a associação entre medidas de prevenção (diagnóstico e atenção ergonômica), de promoção (educação ergonômica) $)^{(8-9,16)}$, modificações na organização do trabalho(11,13,17) e minimização dos riscos ergonômicos $(p<0,005)$. Nos de métodos mistos ${ }^{(15,27-28)}$, evidenciaram-se a identificação da organização e dos determinantes da atividade de trabalho(28), do diário de tarefas ${ }^{(15,28)}$ e da divisão do trabalho em leve e pesado ${ }^{(27)}$ como estratégias promissoras para a minimização 
dos riscos ergonômicos.

Quanto à força de evidência(23) , predominaram estudos de intervenção com evidências de nível 2 ( $n=11 ; 52 \%)^{(3,5-7,9,12,14,18,24-26)}$ e nível 3 (um; 5\%)(4). Sequencialmente, estudos diagnósticos (nível 6) ${ }^{(8,10,11,13,15-17,27-28)}$ (nove; 43\%) e prognósticos (nível 4) ${ }^{(8,10)}$ (dois; 9,5\%).

\section{DISCUSSÃO}

\section{Caracterização dos riscos ergonômicos em trabalhadores de limpeza}

As pesquisas sobre os riscos ergonômicos constituem uma tendência dos últimos seis anos (2012 a 2018) $)^{(2-3,5-13,16-17,20,24-26,28-31)}$. Contudo, no Brasil, observam-se poucos estudos com trabalhadores de limpeza ${ }^{(1-2,11,20,29,31)}$, refletindo um déficit de reconhecimento e valorização ${ }^{(24,31)}$ e a sua invisibilidade no contexto hospitalar(5,31) e institucional(24).

Predominaram estudos com os trabalhadores de limpeza hospitalar ${ }^{(5,7,10,13-16,24,26-27)}$, evidenciando uma elevada exposição aos riscos ergonômicos, altas demandas físicas, movimentos repetitivos, altas cargas estáticas musculares e posturas inadequadas ${ }^{(17)}$. As consequências incluem a elevada prevalência de distúrbios osteomusculares e carga de trabalho (18), com impacto negativo na qualidade de vida. Para as instituições, incluem custos significativos $^{(1)}$ e queda na qualidade dos serviços realizados (faltas, licenças médicas e afastamentos) ${ }^{(15)}$.

Os cenários de atuação dos trabalhadores de limpeza hospitalar são enfermarias, corredores e demais setores intra-hospitalares, realizando atividades leves e pesadas ${ }^{(27)}$. A primeira é caracterizada por posturas neutras, andar, movimentos repetitivos das articulações dos membros superiores empurrando um esfregão de um a seis kg (úmido ou seco). A segunda caracteriza-se por posturas flexionadas, movimentos repetitivos rápidos, envolvendo as articulações dos membros superiores e pesos leves (pó) ou pesos de um a três $\mathrm{kg}$ (esvaziamento de cestos de lixo) ${ }^{(27)}$.

O panorama dos riscos ergonômicos no trabalho de limpeza evidenciou que aspectos ergonômicos negativos estão associados ao desenvolvimento ou agravamento de sintomas musculoesqueléticos ${ }^{(3-8,10-18,26)}$. Essa associação acontece porque as atividades de higienização e limpeza são executadas manualmente, dotadas de esforços repetitivos, com uso de força física e de equipamentos não adaptados às necessidades psicofisiológicas do trabalhador, somando-se ao descanso insuficiente, turnos e carga de trabalho mal distribuídos ${ }^{(8,12)}$. Como resultado, tem-se acidentes de trabalho, diminuição da capacidade funcional, implicações na qualidade de vida e DORT ${ }^{(3-8,10-18,26)}$.

Em estudo, a prevalência de DME em trabalhadores de limpeza hospitalar, nos últimos sete dias, foi de 70,1\%(29). Destes, 25,5\% relataram intensidade forte a insuportável. Também constatou-se que aqueles com maior prevalência de DME possuíam menores escores nas dimensões da qualidade de vida ${ }^{(29)}$.

Ademais, os movimentos repetitivos, o esforço físico(8-9,11,13,18,24-26) e a carga física/ postural de trabalho ${ }^{(12,15,17,27)}$ constituem riscos ergonômicos presentes nas atividades de limpeza de ambientes, cômodos e banheiros. Estes resultam da postura inadequada na inclinação para limpar, exigindo flexão lombar; do transporte manual de cargas e do uso de força física nos membros superiores. O esforço físico esteve associado à subestimação da duração das pausas no trabalho, sendo mais elevada entre os trabalhadores de limpeza do que nos de escritório(16).

Evidenciou-se fatores adicionais na exposição aos riscos ergonômicos, dentre eles: organizacionais ${ }^{(17-18,24,27)}$, clima de trabalho ${ }^{(24)}$ e psicossociais ${ }^{(18,28)}$. Para tanto, as modificações 
na organização do trabalho devem ser ampliadas, priorizando o desenvolvimento de competências $^{(4)}$, dimensionamento ${ }^{(24)}$, rotação de tarefas ${ }^{(24)}$, redesenho da atribuição de tarefas por sexo(27); planejamento e execução de pausas de trabalho(13-17,24) e manutenção de áreas de reposição de materiais em cada andar ${ }^{(11)}$. Assim, possibilita-se a redução da carga física de trabalho e o alívio de $\mathrm{DME}^{(11,24)}$.

Quanto aos fatores psicossociais do trabalho(18,28), eles são definidos como a interação entre o conteúdo do trabalho, condições organizacionais e ambientais, as competências e necessidades individuais e do trabalhador ${ }^{(30)}$. Estas interações podem propiciar estresse ${ }^{(24)}$, falta de reconhecimento ${ }^{(24)}$, desvalorização ${ }^{(24)}$, desigualdade de gênero ${ }^{(28)}$, déficit de suporte social(18), a depender da forma como os trabalhadores de limpeza as experienciam.

\section{Estratégias para minimização dos riscos ergonômicos em trabalhadores de limpeza}

Esta síntese de evidências recomenda a utilização da educação ergonômica como uma estratégia efetiva, principalmente relacionada aos sintomas musculoesqueléticos ${ }^{(3-8,10-18,24)}$. Esta estratégia mostrou-se eficaz na redução do absenteísmo relacionado à ocorrência de DORT ${ }^{(4)}$; no menor nível de atividade muscular do trapézio de ambos os lados $(p=0,03)$, na menor amplitude de movimento e velocidade angular do tronco e menor carga cardiovascular $(p=0,02)^{(8)}$; na eliminação de perigos do processo de trabalho ${ }^{(10)}$ e na redução de DORT ${ }^{(16)}$.

Evidenciou-se que a educação ergonômica facilita a mudança de comportamento dos trabalhadores, mediante ações de autocuidado ${ }^{(28)}$. Ela pode ser realizada por meio de instruções verbais e visuais sobre ergonomia(10); educação em saúde sobre posturas corporais adequadas e manuseio de equipamentos ${ }^{(17)}$; massagens; atividades fitness, entre outras $^{(10)}$.

Quanto aos efeitos das orientações sobre ergonomia (9-10,17), a respeito da rotina diária de limpeza (varrimento, mudança de lixeiras e limpeza de superfícies), observaram-se múltiplos benefícios musculoesqueléticos e cardiovasculares ${ }^{(9)}$. Destacam-se: redução da carga geral de trabalho, indução a um padrão mais complexo de atividade muscular, menor amplitude de movimento e velocidade angular do tronco, menor carga cardiovascular ${ }^{(9)}$ e aumento da conscientização sobre cuidados individuais ${ }^{(10)}$.

Quanto aos equipamentos (máquinas lavadoras e extratoras, aspiradores, rodos, baldes, panos, espremedores, polidoras, discos abrasivos e carros para transporte de resíduos)(32), a Agência Nacional de Vigilância Sanitária (ANVISA) não especifica quais ações preventivas, no contexto da ergonomia, podem ser adotadas na utilização desses equipamentos ${ }^{(32)}$. Esta síntese recomenda modificações com a utilização de equipamentos com melhor design ergonômico e layout interfuncional $(6,11,13,17,24,26)$. Incluem: cabos de eixo longo ou carrinho de mão $(p<0,001)^{(6)}$; utilização do aspirador em máquina do tipo caixa $(p=0,011)$ comparado à máquina tipo mochila $(p=0,016)^{(13)}$, diminuição do peso dos carros funcionais e de transportes de resíduos ${ }^{(11)}$ e esfregão com a altura do cabo ajustável(24,26).

As evidências indicam o mop (esfregão) como o equipamento de trabalho prevalente na limpeza $a^{(9,11,24,26)}$. Recomenda-se a otimização do uso e um cabo facilmente ajustável ${ }^{(26)}$. Tal medida pode ser efetiva na redução da demanda muscular, decorrente do uso excessivo dos músculos do ombro ou do queixo, em comparação com o nível dos olhos $(p<0,001)^{(26)}$ e em comparação com o esfregão não ajustável para o músculo deltoide direito $(p=0,003)$; músculo trapézio direito $(p=0,000)$ e extensores de punho esquerdo $(p=0,004)$, obtendo-se um nível médio de conforto de 4,93 e de menor esforço ${ }^{(24)}$. Nesse sentido, uma abordagem holística é necessária para melhorar os benefícios das ferramentas e técnicas no trabalho de limpeza, visto que a implementação e contextualização do esfregão são importantes ${ }^{(11)}$.

A estratégia de realização de exercício aeróbico $^{(5,25)}$ foi realizada em quatro ${ }^{(5)}$ e 12 meses ${ }^{(5,25)}$, mostrando-se eficaz para: aumento na capacidade para o trabalho de 0,59 $\pm 0,27$ pontos (IC 95\%; 0,05-1,13, p=0,03) $)^{(25)}$, diminuição da necessidade de recuperação(25), 
produtividade, identificação da organização e determinantes da atividade de trabalho(5). Quando realizado em quatro meses, o exercício aeróbico foi efetivo para reduzir significativamente (>30\%), no grupo exercício, a intensidade da DME no pescoço, ombros, braços/punhos ${ }^{(5)}$. Essa tendência foi mantida nos 12 meses seguintes $(p=0,04)$.

No entanto, como um efeito não intencional, o exercício físico proporcionou o aumento de intensidade da dor nas extremidades inferiores ${ }^{(5)}$ e não obteve efeito na percepção do esforço percebido e na produtividade, com tendência para maior efeito do exercício aeróbico entre os trabalhadores mais jovens ${ }^{(25)}$. Por isso, recomenda-se que os exercícios aeróbicos sejam realizados como parte integrante da jornada de trabalho(25), com adaptação do exercício, de forma a manter apenas o efeito positivo sobre a DME(5).

Como limitação desta revisão, elenca-se a dificuldade em comparar os resultados relacionados aos riscos ergonômicos, antes e após a realização das estratégias, visto a heterogeneidade em relação à apresentação dos resultados; bem como, de parâmetros e escalas.

\section{CONCLUSÃO}

Esta síntese de evidências recomenda a utilização de estratégias de educação ergonômica, modificações nas ferramentas de trabalho, realização de exercício aeróbico, método para avaliação de risco, protocolo de vigilância em ergonomia, diário de tarefas; bem como a contextualização com os fatores organizacionais e psicossociais do trabalho.

As estratégias evidenciadas mostraram-se eficazes e poderão contribuir para o campo da saúde na promoção e recuperação de acometimentos físicos dos trabalhadores de limpeza.

\section{AGRADECIMENTOS}

Ao Programa de Bolsas de Iniciação Científica (PROBIC/FAPERGS - Edital 034/2019) e à Coordenação de Aperfeiçoamento de Pessoal de Nível Superior - Brasil (CAPES) Código de Financiamento 001.

\section{REFERÊNCIAS}

1. Silva LG da, Haddad M do CL, Domansky R de C, Vituri DW. Capacidade para o trabalho entre trabalhadores de higiene e limpeza de um hospital universitário público. Rev. Eletr. Enf. [Internet]. 2010 [acesso em 12 nov 2019]; 12(1). Disponível em: https://doi.org/10.5216/ree.v12i1.5788.

2. Chaves LDP, Camelo SHH, Silva MR, Silva NM, Pereira AP. Hospital governance, hygiene and cleaning: nurse management space. Texto contexto-enferm. [Internet]. 2015 [acesso em 21 set 2019]; 24(4). Disponível em: http://dx.doi.org/10.1590/0104-0707201500004010014.

3. Dahlqvist C, Nordander C, Forsman M, Enquist H. Self-recordings of upper arm elevation during cleaning - comparison between analyses using a simplified reference posture and a standard reference posture. BMC Musculoskeletal Disorders. [Internet]. 2018 [acesso em 14 ago 2019]; 19(402). Disponível 
em: http://doi.org/10.1186/s12891-018-2328-8.

4. Landstad BJ, Gelin G, Malmquist C, Vinberg S. A statistical human resources costing and accounting model for analysing the economic effects of an intervention at a workplace. Ergonomics. [Internet]. 2002 [acesso em 21 ago 2019]; 45(11). Disponível em: https://doi.org/10.1080/00140130210136053.

5. Korshoj M, Jorgensen MB, Lidegaard M, Mortensen OS, Krustrup P, Holtermann A, et al. Decrease in musculoskeletal pain after 4 and 12 months of an aerobic exercise intervention: a worksite RCT among cleaners. Scand J Public Health [Internet]. 2017 [acesso em 22 ago 2019]; 46(1). Disponível em: https:// doi.org/10.1177/1403494817717833.

6. Lofqvist L, Osvalder AL, Bligård LO, Pinzke S. An analytical ergonomic risk evaluation of body postures during daily cleaning tasks in horse stables. Work. [Internet]. 2015 [acesso em 23 set 2019]; 51(4).

Disponível em: https://doi.org/10.3233/WOR-152022.

7. Jonker D, Gustafsson E, Rolander B, Arvidsson I, Nordander C. Health surveillance under adverse ergonomics conditions - validity of a screening method adapted for the occupational health service. Ergonomics [Internet]. 2015 [acesso em 23 set 2019]; 58(9). Disponível em: https://doi.org/10.1080/00140 139.2015.1019575.

8. Habib MM, Rahman SU. Musculoskeletal symptoms (MSS) and their associations with ergonomic physical risk factors of the women engaging in regular rural household activities: A picture from a rural village in Bangladesh. Work [Internet]. 2015 [acesso em 23 set 2019]; 50(3). Disponível em: https://doi. org/10.3233/WOR-151990.

9. Samani A, Holtermann A, Søgaard K, Holtermann A, Madeleine P. Following ergonomics guidelines decreases physical and cardiovascular workload during cleaning tasks. Ergonomics [Internet]. 2012 [acesso em 25 set 2019]; 55(3). Disponível em: https://doi.org/10.1080/00140139.2011.640945.

10. Missar VJ, Metcalfe D, Gilmore G. Transforming a hospital safety and ergonomics Program: a four year journey of change. Work [Internet]. 2012 [acesso em 25 set 2019]; 41(suppl1). Disponível em: https://doi. org/10.3233/WOR-2012-0988-5912.

11. Silva-Junior JS, Correa LRC, Morrone LC. Evaluation of lumbar overload in hotel maids. Work [Internet]. 2012 [acesso em 29 set 2019]; 41(supl1). Disponível em: https://doi.org/10.3233/WOR-20120488-2496.

12. Madeleine P, Søgaard K, Holtermann A, Samani A. Level of self-reported neck/shoulder pain and biomechanical workload in cleaners. Work [Internet]. 2012 [acesso em 28 set 2019]; 41(supl1). Disponível em: https://doi.org/10.3233/WOR-2012-0195-447.

13. Bell AF, Steele JR. Risk of musculoskeletal injury among cleaners during vacuuming. Ergonomics [Internet]. 2012 [acesso em 28 set 2019]; 55(2). Disponível em: https://doi.org/10.1080/00140139.2011.59 2605.

14. Mathiassen SE, Nordander C, Svendsen SW, Wellman HM, Dempsey PG. Task-based estimation of mechanical job exposure in occupational groups. Scand J Work Environ Health [Internet]. 2005 [acesso em 29 set 2019]; 31(2). Disponível em: https://doi.org/10.5271/sjweh.861.

15. Unge J, Hansson GA, Ohlsson K, Nordander C, Axmon A, Winkel J, et al. Validity of self-assessed reports of occurrence and duration of occupational tasks. Ergonomics [Internet]. 2005 [acesso em 29 set 2019]; 48(1). Disponível em: https://doi.org/10.1080/00140130412331293364.

16. Joseph B, Naveen R, Suguna A, Surekha A. Prevalence, Pattern and Factors Associated with Workrelated Musculoskeletal Disorders (WRMD) among Housekeeping Workers in a Private Tertiary Care Hospital in Bangalore. J Health Manag [Internet]. 2016 [acesso em 01 out 2019]; 18(4). Disponível em: https://doi.org/10.1177/0972063416666151.

17. Siqueira CE, Roche AG. Occupational health profile of Brazilian immigrant housecleaners in Massachusetts. New Solutions [Internet]. 2014 [acesso em 01 set 2019]; 23(3). Disponível em: https://doi. org/10.2190/NS.23.3.e. 
18. Unge J, Ohlsson K, Nordander C, Hansson GA, Skerving S, Balogh I. Differences in physical workload, psychosocial factors and musculoskeletal disorders between two groups of female hospital cleaners with two diverse organizational models. Int Arch Occup Environ Health [Internet]. 2007 [acesso em 21 set 2019]; 81. Disponível em: http://doi.org/10.1007/s00420-007-0208-x.

19. Ministério do Trabalho (BR). Normas Regulamentadoras: Segurança e Medicina do Trabalho. São Paulo: Atlas; 2013.

20. Souza R de S, Cortez EA, Carmo TG do, Santana RF. Occupational diseases of workers cleaning service in hospital environment: educational proposal to minimize exposure. Enferm. glob. [Internet]. 2016 [acesso em 06 nov 2019]; 15(42). Disponível em: http://scielo.isciii.es/pdf/eg/v15n42/en revision5.pdf.

21. Mendes KDS, Silveira RC de CP, Galvão CM. Revisão integrativa: método de pesquisa para a incorporação de evidências na saúde e na enfermagem. Texto contexto-enferm. [Internet]. 2008 [acesso em 12 nov 2019]; 17(4). Disponível em: http://dx.doi.org/10.1590/S0104-07072008000400018.

22. Santos CM da C, Pimenta CA de M, Nobre MRC. The PICO strategy for the research question construction and evidence search. Rev Latino-Am Enfermagem. [Internet]. 2007 [acesso em 02 set 2019]; 15(3). Disponível em: http://dx.doi.org/10.1590/S0104-11692007000300023.

23. Melnyk BM, Fineout-Overholt E. Making the case for evidence-based practice and cultivating a spirit of inquiry. In: Melnyk BM, Fineout-Overholt. Evidence-based practice in nursing \& healthcare. A guide to best practice. Philadelphia: Wolters Kluwer, Lippincott Williams \& Wilkins; 2011. p.3-24.

24. Öhrling T, Kumar R, Abrahamsson L. Assessment of the development and implementation of tools in contract cleaning. Appl Ergon [Internet]. 2012 [acesso em 24 set 2019]; 43(4). Disponível em: https://doi. org/10.1016/j.apergo.2011.11.006.

25. Lidegaard M, Sogaard K, Krustrup P, Holtermann A, Korshoj M. Effects of 12 months aerobic exercise intervention on work ability, need for recovery, productivity and rating of exertion among cleaners: a worksite RCT. Int Arch Occup Environ Health. [Internet]. 2018 [acesso em 29 set 2019]; 91(2). Disponível em: https://doi.org/10.1007/s00420-017-1274-3.

26. Wallius MA, Rissanen SM, Bragge T, Vartiainen P, Karjalainen PA, Räsänen K, et al. Effects of mop handle height on shoulder muscle activity and perceived exertion during floor mopping using a figure eight method. Industrial Health [Internet]. 2016 [acesso em 29 set 2019]; 54(1). Disponível em: https://doi. org/10.2486/indhealth.2015-0108.

27. Messing K, Chatigny C, Courville J. "Light" and "heavy" work in the housekeeping service of a hospital. Appl Ergon [Internet]. 1998 [acesso em 29 set 2019]; 29(6). Disponível em: https://doi. org/10.1016/S0003-6870(98)00013-1.

28. Lefrançois M, Saint-Charles J, Riel J. Work/Family balancing and 24/7 work schedules: network analysis of strategies in a transport company cleaning service. New Solutions: a J Envir Occup Health Policy [Internet]. 2017 [acesso em 28 set 2019]; 27(3). Disponível em: https://doi. org/10.1177/1048291117725718.

29. Luz EMF da, Magnago TSB de S, Greco PBT, Ongaro JD, Lanes TC, Lemos JC. Prevalence and factors associated with musculoskeletal pain in hospital cleaning workers. Texto contexto-enferm. [Internet]. 2017 [acesso em 05 dez 2019]; 26(2). Disponível em: http://dx.doi.org/10.1590/0104-07072017000870016.

30. Fernandes C, Pereira A. Exposure to psychosocial risk factors in the context of work: a systematic review. Rev Saude Publica. [Internet]. 2016 [acesso em 12 dez 2019]; 50. Disponível em: http://dx.doi. org/10.1590/S1518-8787.2016050006129.

31. Lanza KL, Ribeiro BG de A, Martins JT, Okubo CVC, Galdino MJQ, Robazzi ML do CC. Feelings of workers of the cleaning staff of a teaching hospital. Cogitare enferm. [Internet]. 2019 [acesso em $12 \mathrm{dez}$ 2019]; 24. Disponível em: https://dx.doi.org/10.5380/ce.v24i0.62467.

32. Agência Nacional de Vigilância Sanitária (BR). Segurança do paciente em serviços de saúde: limpeza e 
desinfecção de superfícies. Brasília: Anvisa; 2012.

COMO REFERENCIAR ESTE ARTIGO:

Luz EMF da, Munhoz OL, Morais BX, Silva SC da, Almeida FO, Magnago TSB de S. Estratégias para minimizar os riscos ergonômicos em trabalhadores de limpeza: revisão integrativa. Cogitare enferm. [Internet]. 2021 [acesso em "colocar data de acesso, dia, mês abreviado e ano"]; 26. Disponível em: http://dx.doi.org/10.5380/ ce.v26i0.71073.

*Artigo extraído da tese de doutorado "Riscos ergonômicos no Serviço Hospitalar de Limpeza: estudo convergente assistencial para a prevenção de dor musculoesquelética". Universidade Federal de Santa Maria, 2019.

Recebido em: 06/01/2020

Aprovado em: 09/09/2020

Autor Correspondente:

Emanuelli Mancio Ferreira da Luz

Universidade Federal de Santa Maria - Santa Maria, RS, Brasil

E-mail: manumfluz@gmail.com

Contribuição dos autores:

Contribuições substanciais para a concepção ou desenho do estudo; ou a aquisição, análise ou interpretação de dados do estudo - EMFL, OLM, BXM, SCS, FOA

Elaboração e revisão crítica do conteúdo intelectual do estudo - TSBBSM

Aprovação da versão final do estudo a ser publicado - TSBBSM

Responsável por todos os aspectos do estudo, assegurando as questões de precisão ou integridade de qualquer parte do estudo - EMFL

Copyright $\odot 2021$ Este é um artigo em acesso aberto distribuído nos termos da Licença Creative Commons Atribuição, que permite o uso irrestrito, a distribuição e reprodução em qualquer meio desde que $\circ$ artigo original seja devidamente citado. 